\title{
Existence of solutions for fractional differential equations with integral boundary conditions
}

\author{
Rian Yan, Shurong Sun ${ }^{*}$, Hongling Lu and Yan Zhao
}

\author{
"Correspondence: \\ sshrong@163.com \\ School of Mathematical Sciences, \\ University of Jinan, Jinan, Shandong \\ 250022, PR China
}

\begin{abstract}
In this paper, we study boundary-value problems for the following nonlinear fractional differential equations involving the Caputo fractional derivative: ${ }^{C} D_{0+}^{\alpha} x(t)=f\left(t, x(t),{ }^{C} D_{0+}^{\beta} x(t)\right), t \in[0,1], x(0)+x^{\prime}(0)=y(x), \int_{0}^{1} x(t) d t=m$, $x^{\prime \prime}(0)=x^{\prime \prime \prime}(0)=\cdots=x^{(n-1)}(0)=0$, where ${ }^{C} D_{0+1}^{\alpha}{ }^{c} D_{0+}^{\beta}$ are the Caputo fractional derivatives, $f:[0,1] \times \mathbb{R} \times \mathbb{R} \rightarrow \mathbb{R}$ is a continuous function, $y: C([0,1], \mathbb{R}) \rightarrow \mathbb{R}$ is a continuous function and $m \in \mathbb{R}, n-1<\alpha<n(n \geq 2), 0<\beta<1$ is a real number. By means of the Banach fixed-point theorem and the Schauder fixed-point theorem, some solutions are obtained, respectively. As applications, some examples are presented to illustrate our main results.

MSC: $34 \mathrm{~A} 08 ; 34 \mathrm{~B} 10$

Keywords: fractional differential equation; boundary-value problem; fixed-point theorem
\end{abstract}

\section{Introduction}

Fractional differential equations have been of increasing importance in the past decades due to their diverse applications in science and engineering, such as the memory of a variety of materials, signal identification and image processing, optical systems, thermal system materials and mechanical systems, control system, etc., see [1, 2]. Many interesting results on the existence of solutions of various classes of fractional differential equations have been obtained, see [3-17], and the references therein.

Recently, much attention has been focused on the study of the existence and multiplicity of solutions or positive solutions for boundary-value problems of fractional differential equations with local boundary-value problems by the use of techniques of nonlinear analysis (fixed-point theorems, Leray-Schauder theory, the upper and lower solution method, etc.), see [7-17].

On the other hand, integer-order differential equations boundary-value problems with integral boundary conditions arise in a variety of different areas of applied mathematics and physics. For example, blood flow problems, chemical engineering, thermo-elasticity, underground water flow, population dynamics, and so forth can be reduced to nonlocal problems with integral boundary conditions. For a detailed description of the integral boundary conditions, we refer the reader to some recent papers [18-20] and the references therein.

o2014 Yan et al.: licensee Springer. This is an Open Access article distributed under the terms of the Creative Commons Attribution License (http://creativecommons.org/licenses/by/2.0), which permits unrestricted use, distribution, and reproduction in any medium, provided the original work is properly cited. 
In fact, there we have the same requirements for fractional differential equations. Boundary-value problems for fractional-order differential equations with nonlocal boundary conditions constitute a very interesting and important class of problems. This type of boundary-value problems has been investigated in [21-24]. Lately, Zhang et al. [25] investigated the existence of solutions for a fractional nonlinear integro-differential equation of mixed type on a semi-infinite interval in a Banach space E. Li et al. [26] studied the existence and uniqueness of a positive solution for nonlinear fractional differential equations. Anguraj et al. [27] obtained new existence results for fractional integro-differential equations with impulsive and integral conditions.

There were several definitions of fractional derivatives such as Riemann-Liouville, Caputo, Weyl, etc. Applied problems require those definitions of fractional derivatives that allow the utilization of physically interpretable initial and boundary conditions. The Caputo fractional derivative fulfills these requirements.

Cabada et al. investigated the existence of positive solutions of the following nonlinear fractional differential equations with integral boundary-value conditions [22]:

$$
\begin{aligned}
& { }^{C} D^{\alpha} u(t)+f(t, u(t))=0, \quad 0<t<1, \\
& u(0)=u^{\prime \prime}(0)=0, \quad u(1)=\lambda \int_{0}^{1} u(s) d s,
\end{aligned}
$$

where $2<\alpha<3,0<\lambda<2,{ }^{C} D^{\alpha}$ is the Caputo fractional derivative and $f:[0,1] \times[0, \infty) \rightarrow$ $[0, \infty)$ is a continuous function.

In 2013, Vong et al. studied the existence of positive solutions of a class of singular fractional differential equations with nonlocal boundary conditions [28],

$$
\begin{aligned}
& { }^{C} D_{0+}^{\alpha} u(t)+f(t, u(t))=0, \\
& u^{\prime}(0)=\cdots=u^{(n-1)}=0, \quad u(1)=\int_{0}^{1} u(s) d \mu(s),
\end{aligned}
$$

where $n \geq 2, \alpha \in(n-1, n)$, and $\mu(s)$ is a function of bounded variation, $f$ may have a singularity at $t=1$, and $\int_{0}^{1} d \mu(s)<1$.

Agarwal et al. investigated the existence of solutions for the singular fractional boundary-value problems [29]

$$
\begin{aligned}
& D^{\alpha} u(t)+f\left(t, u(t), D^{\mu} u(t)\right)=0, \\
& u(0)=u(1)=0,
\end{aligned}
$$

where $1<\alpha<2,0<\mu \leq \alpha-1$ are real numbers, $D^{\alpha}$ is the standard Rieman-Liouville fractional derivative, $f$ satisfies the Caratheodory conditions on $[0,1] \times \mathbb{B}, \mathbb{B}=(0, \infty) \times \mathbb{R}$ $(f \in \operatorname{Car}([0,1] \times \mathbb{B})), f$ is positive, and $f(t, x, y)$ is singular at $x=0$.

Benchohra et al. studied the boundary-value problem for the fractional differential equations with nonlocal conditions [30]

$$
\begin{aligned}
& { }^{C} D_{0+}^{\alpha} y(t)=f(t, y(t)), \quad t \in J=[0, T], 1<\alpha \leq 2, \\
& y(0)=g(y), \quad y(T)=y_{T},
\end{aligned}
$$


where ${ }^{C} D_{0+}^{\alpha}$ is the Caputo fractional derivative, $f:[0, T] \times \mathbb{R} \rightarrow \mathbb{R}$ is a continuous function, $g: C(J, \mathbb{R}) \rightarrow \mathbb{R}$ is a continuous function and $y_{T} \in \mathbb{R}$.

Motivated by all the works above, this paper deals with the existence and uniqueness of solutions for the boundary-value problem of the fractional differential equations

$$
\begin{aligned}
& { }^{C} D_{0+}^{\alpha} x(t)=f\left(t, x(t),{ }^{C} D_{0+}^{\beta} x(t)\right), \quad t \in[0,1], \\
& x(0)+x^{\prime}(0)=y(x), \quad \int_{0}^{1} x(t) d t=m, \\
& x^{\prime \prime}(0)=x^{\prime \prime \prime}(0)=\cdots=x^{(n-1)}(0)=0,
\end{aligned}
$$

where ${ }^{C} D_{0+}^{\alpha},{ }^{C} D_{0+}^{\beta}$ are the Caputo fractional derivatives, $f:[0,1] \times \mathbb{R} \times \mathbb{R} \rightarrow \mathbb{R}$ is a continuous function, $y: C([0,1], \mathbb{R}) \rightarrow \mathbb{R}$ is a continuous function, and $m \in \mathbb{R}, n-1<\alpha<n$ $(n \geq 2), 0<\beta<1$ is a real number.

The paper is organized as follows. In Section 2, we shall introduce some definitions and lemmas to prove our main results. In Section 3, we establish some criteria for the existence for the boundary problem (1.1) with nonlocal boundary conditions (1.2) and (1.3) by using the Banach fixed-point theorem and the Schauder fixed-point theorem. Finally, we present three examples to illustrate our main results.

\section{Preliminaries}

In this section, we introduce notations and definitions of fractional calculus, and we prove a lemma before stating our main results.

Let $X=\left\{x: x \in C[0,1], D^{\beta} x \in C[0,1]\right\}$. We define $\|x\|=\max _{t \in[0,1]}|x(t)|,\|x\|_{1}=\max \{\|x\|$, $\left.\left\|D^{\beta} x\right\|\right\}$; then $\left(X,\|\cdot\|_{1}\right)$ is a Banach space.

Definition 2.1 ([2]) For a continuous function $y:(0, \infty) \rightarrow \mathbb{R}$, the Riemann-Liouville fractional integral of order $\alpha$ is defined as

$$
I_{0+}^{\alpha} y(t)=\frac{1}{\Gamma(\alpha)} \int_{0}^{t}(t-s)^{\alpha-1} y(s) d s, \quad \alpha>0,
$$

provided the right side integral is pointwise defined on $[0, \infty)$.

Definition 2.2 ([2]) The Caputo fractional derivative of order $\alpha$ for a continuous function $y(t)$ is defined by

$$
C_{D_{0+}}^{\alpha} y(t)=\frac{1}{\Gamma(n-\alpha)} \int_{0}^{t}(t-s)^{n-\alpha-1} y^{(n)}(s) d s, \quad \alpha>0
$$

where $\Gamma$ is the Gamma function, $n=[\alpha]+1$, and $[\alpha]$ denotes the integer part of number $\alpha$, and provided the right side integral is pointwise defined on $[0, \infty)$.

Lemma 2.1 ([2]) Let $u \in C^{m}[0, T]$ and $q \in(m-1, m], m \in N$. Then for $t \in[0, T]$,

$$
I^{q C} D_{0+}^{q} u(t)=u(t)-\sum_{k=0}^{m-1} \frac{t^{k}}{k !} u^{(k)}(0) .
$$


Lemma 2.2 Let $n-1<\alpha<n$; if $x \in C^{n}[0,1]$ is a solution of the following fractional differential equations:

$$
\begin{aligned}
& { }^{C} D_{0+}^{\alpha} x(t)=h(s), \quad t \in[0,1], \\
& x(0)+x^{\prime}(0)=y(x), \quad \int_{0}^{1} x(t) d t=m, \\
& x^{\prime \prime}(0)=x^{\prime \prime \prime}(0)=\cdots=x^{(n-1)}(0)=0,
\end{aligned}
$$

then $x(t)$ can be represented by

$$
\begin{aligned}
x(t)= & \frac{1}{\Gamma(\alpha)} \int_{0}^{t}(t-s)^{\alpha-1} h(s) d s+2(1-t) m+(2 t-1) y(x) \\
& +\frac{2(t-1)}{\Gamma(\alpha+1)} \int_{0}^{1}(1-s)^{\alpha} h(s) d s .
\end{aligned}
$$

Proof By Lemma 2.1 and the boundary conditions $x^{\prime \prime}(0)=x^{\prime \prime \prime}(0)=\cdots=x^{(n-1)}(0)=0$, we have

$$
\begin{aligned}
x(t) & =I_{0+}^{\alpha} h(t)+x(0)+x^{\prime}(0) t+\frac{x^{\prime \prime}(0)}{2 !} t^{2}+\cdots+\frac{x^{(n-1)}(0)}{(n-1) !} t^{n-1} \\
& =\frac{1}{\Gamma(\alpha)} \int_{0}^{t}(t-s)^{\alpha-1} h(s) d s+x(0)+x^{\prime}(0) t .
\end{aligned}
$$

Hence

$$
\int_{0}^{1} x(t) d t=\int_{0}^{1} \int_{0}^{t} \frac{(t-s)^{\alpha-1}}{\Gamma(\alpha)} h(s) d s d t+x(0)+\frac{1}{2} x^{\prime}(0) .
$$

By the boundary conditions $x(0)+x^{\prime}(0)=y(x), \int_{0}^{1} x(t) d t=m$, we obtain

$$
x(0)=2 m-y(x)-\frac{2}{\Gamma(\alpha+1)} \int_{0}^{1}(1-s)^{\alpha} h(s) d s
$$

and

$$
x^{\prime}(0)=\frac{2}{\Gamma(\alpha+1)} \int_{0}^{1}(1-s)^{\alpha} h(s) d s+2 y(x)-2 m .
$$

Consequently

$$
\begin{aligned}
x(t)= & \frac{1}{\Gamma(\alpha)} \int_{0}^{t}(t-s)^{\alpha-1} h(s) d s+2(1-t) m+(2 t-1) y(x) \\
& +\frac{2(t-1)}{\Gamma(\alpha+1)} \int_{0}^{1}(1-s)^{\alpha} h(s) d s .
\end{aligned}
$$

Lemma 2.3 (Schauder's fixed point theorem) Let $(E, d)$ be a complete metric space, let $U$ be a closed convex subset of $E$, and let $A: U \rightarrow U$ be a mapping such that the set $\{A u: u \in U\}$ is relatively compact in $E$. Then $A$ has at least one fixed point. 


\section{Main results}

Now we are in the position to establish the main results.

\section{Theorem 3.1 Assume that:}

(H1) There exists a constant $l>0$ such that

$$
\begin{aligned}
& \left|f\left(t, x_{1}, z_{1}\right)-f\left(t, x_{2}, z_{2}\right)\right| \leq l\left(\left|x_{1}-x_{2}\right|+\left|z_{1}-z_{2}\right|\right), \text { for each } t \in[0, T] \text { and all } \\
& x_{1}, x_{2}, z_{1}, z_{2} \in \mathbb{R} .
\end{aligned}
$$

(H2) There exists a constant $l_{1}>0$ such that $\left|y\left(x_{1}\right)-y\left(x_{2}\right)\right| \leq l_{1}\left\|x_{1}-x_{2}\right\|$, for each $x_{1}, x_{2} \in C([0, T], \mathbb{R})$.

(H3) $\theta=\max \left\{\left(\frac{2}{\Gamma(\alpha+1)}+\frac{4}{\Gamma(\alpha+2)}\right) l+l_{1}, \frac{1}{\Gamma(2-\beta)}\left[\left(\frac{2}{\Gamma(\alpha)}+\frac{4}{\Gamma(\alpha+2)}\right) l+2 l_{1}\right]\right\}<1$.

Then the BVP (1.1)-(1.3) has a unique solution.

Proof Transform the BVP (1.1)-(1.3) into a fixed-point problem. Consider the operator

$$
F: X \rightarrow X
$$

defined by

$$
\begin{aligned}
F(x)(t)= & \frac{1}{\Gamma(\alpha)} \int_{0}^{t}(t-s)^{\alpha-1} f\left(s, x(s),{ }^{C} D^{\beta} x(s)\right) d s+2(1-t) m+(2 t-1) y(x) \\
& +\frac{2(t-1)}{\Gamma(\alpha+1)} \int_{0}^{1}(1-s)^{\alpha} f\left(s, x(s),{ }^{C} D^{\beta} x(s)\right) d s .
\end{aligned}
$$

Clearly, the fixed points of the operator $F$ are solutions of the problem (1.1)-(1.3).

Let $x_{1}, x_{2} \in X$. Then

$$
\begin{aligned}
& \left|F\left(x_{1}\right)(t)-F\left(x_{2}\right)(t)\right| \\
& \leq \frac{1}{\Gamma(\alpha)} \int_{0}^{t}(t-s)^{\alpha-1}\left|f\left(s, x_{1}(s),{ }^{C} D^{\beta} x_{1}(s)\right)-f\left(s, x_{2}(s),{ }^{C} D^{\beta} x_{2}(s)\right)\right| d s \\
& \quad+\frac{2(1-t)}{\Gamma(\alpha+1)} \int_{0}^{1}(1-s)^{\alpha}\left|f\left(s, x_{1}(s),{ }^{C} D^{\beta} x_{1}(s)\right)-f\left(s, x_{2}(s),{ }^{C} D^{\beta} x_{2}(s)\right)\right| d s \\
& \quad+\left|y\left(x_{1}\right)-y\left(x_{2}\right)\right| .
\end{aligned}
$$

Consider the conditions ( $\mathrm{H} 1)$ and (H2), implying that

$$
\begin{aligned}
& \left|f\left(t, x_{1}(t),{ }^{C} D^{\beta} x_{1}(t)\right)-f\left(t, x_{2}(t),{ }^{C} D^{\beta} x_{2}(t)\right)\right| \\
& \quad \leq l\left(\left|x_{1}(t)-x_{2}(t)\right|+\left|{ }^{C} D^{\beta} x_{1}(t)-{ }^{C} D^{\beta} x_{2}(t)\right|\right) \\
& \quad \leq l\left(\left\|x_{1}-x_{2}\right\|+\left\|{ }^{C} D^{\beta} x_{1}-{ }^{C} D^{\beta} x_{2}\right\|\right) \\
& \quad \leq 2 l\left\|x_{1}-x_{2}\right\|_{1}
\end{aligned}
$$

and

$$
\left|y\left(x_{1}\right)-y\left(x_{2}\right)\right| \leq l_{1}\left\|x_{1}-x_{2}\right\| \leq l_{1}\left\|x_{1}-x_{2}\right\|_{1},
$$


thus, we have

$$
\begin{aligned}
\left\|F\left(x_{1}\right)-F\left(x_{2}\right)\right\| \leq & \frac{1}{\Gamma(\alpha)} \int_{0}^{t}(t-s)^{\alpha-1} d s \cdot 2 l\left\|x_{1}-x_{2}\right\|_{1}+l_{1}\left\|x_{1}-x_{2}\right\|_{1} \\
& +\frac{2}{\Gamma(\alpha+1)} \int_{0}^{1}(1-s)^{\alpha} d s \cdot 2 l\left\|x_{1}-x_{2}\right\|_{1} \\
\leq & {\left[\left(\frac{2}{\Gamma(\alpha+1)}+\frac{4}{\Gamma(\alpha+2)}\right) l+l_{1}\right]\left\|x_{1}-x_{2}\right\|_{1} . }
\end{aligned}
$$

As

$$
\begin{aligned}
\left|\left(F x_{1}\right)^{\prime}(t)-\left(F x_{2}\right)^{\prime}(t)\right| & \\
\leq & \frac{1}{\Gamma(\alpha-1)} \int_{0}^{t}(t-s)^{\alpha-2}\left|f\left(s, x_{1}(s),{ }^{C} D^{\beta} x_{1}(s)\right)-f\left(s, x_{2}(s),{ }^{C} D^{\beta} x_{2}(s)\right)\right| d s \\
& +\frac{2}{\Gamma(\alpha+1)} \int_{0}^{1}(1-s)^{\alpha}\left|f\left(s, x_{1}(s),{ }^{C} D^{\beta} x_{1}(s)\right)-f\left(s, x_{2}(s),{ }^{C} D^{\beta} x_{2}(s)\right)\right| d s \\
& +2\left|y\left(x_{1}\right)-y\left(x_{2}\right)\right| \\
\leq & \frac{1}{\Gamma(\alpha-1)} \int_{0}^{t}(t-s)^{\alpha-2} d s \cdot 2 l\left\|x_{1}-x_{2}\right\|_{1}+2 l_{1}\left\|x_{1}-x_{2}\right\|_{1} \\
& +\frac{2}{\Gamma(\alpha+1)} \int_{0}^{1}(1-s)^{\alpha} d s \cdot 2 l\left\|x_{1}-x_{2}\right\|_{1} \\
\leq & {\left[\left(\frac{2}{\Gamma(\alpha)}+\frac{4}{\Gamma(\alpha+2)}\right) l+2 l_{1}\right]\left\|x_{1}-x_{2}\right\|_{1}, }
\end{aligned}
$$

we obtain

$$
\begin{aligned}
\left|{ }^{C} D^{\beta}\left(F x_{1}\right)(t)-{ }^{C} D^{\beta}\left(F x_{2}\right)(t)\right| & \leq \frac{1}{\Gamma(1-\beta)} \int_{0}^{t}(t-s)^{-\beta}\left|\left(F x_{1}\right)^{\prime}(s)-\left(F x_{2}\right)^{\prime}(s)\right| d s \\
& \leq \frac{1}{\Gamma(2-\beta)}\left[\left(\frac{2}{\Gamma(\alpha)}+\frac{4}{\Gamma(\alpha+2)}\right) l+2 l_{1}\right]\left\|x_{1}-x_{2}\right\|_{1} .
\end{aligned}
$$

Consequently

$$
\left\|F x_{1}-F x_{2}\right\|_{1}=\max \left\{\left\|F\left(x_{1}\right)-F\left(x_{2}\right)\right\|,\left\|{ }^{C} D^{\beta}\left(F x_{1}\right)-{ }^{C} D^{\beta}\left(F x_{2}\right)\right\|\right\} \leq \theta\left\|x_{1}-x_{2}\right\|_{1},
$$

then $F$ is a contraction with $\theta<1$. As a consequence of the Banach fixed-point theorem, we deduce that $F$ has a fixed point which is the unique solution of the problem (1.1)-(1.3). The proof is complete.

Next, we will use the Schauder' fixed-point theorem to prove our result. For the sake of convenience, we set

$$
\begin{aligned}
& M=\max _{t \in[0,1]} \mid f\left(t, x(t), C^{\beta} x(t)\left|, \quad M_{1}=\max _{t \in[0,1]}\right| y(x(t)) \mid,\right. \\
& A=\left(\frac{1}{\Gamma(\alpha+1)}+\frac{2}{\Gamma(\alpha+2)}\right) M+M_{1}+2|m|, \\
& B=\left(\frac{1}{\Gamma(\alpha-\beta+1)}+\frac{2}{\Gamma(2-\beta) \Gamma(\alpha+2)}\right) M+\frac{2}{\Gamma(2-\beta)}\left(M_{1}+|m|\right) .
\end{aligned}
$$


Theorem 3.2 Assume $f:[0,1] \times \mathbb{R} \times \mathbb{R} \rightarrow \mathbb{R}$ is continuous, $y:(C[0,1], \mathbb{R}) \rightarrow \mathbb{R}$ is continuous. Then the BVP (1.1)-(1.3) has a solution.

Proof Let $E=\left\{x: x \in X,\|x\|_{1} \leq r\right\}$, where $r=\max \{A, B\}$. First, we prove that $F: E \rightarrow E$. In fact, for each $t \in[0,1]$, we have

$$
\begin{aligned}
|F(x)(t)| \leq & \frac{1}{\Gamma(\alpha)} \int_{0}^{t}(t-s)^{\alpha-1}\left|f\left(s, x(s),{ }^{C} D^{\beta} x(s)\right)\right| d s+2|m|+|y(x)| \\
& +\frac{2}{\Gamma(\alpha+1)} \int_{0}^{1}(1-s)^{\alpha}\left|f\left(s, x(s),{ }^{C} D^{\beta} x(s)\right)\right| d s \\
\leq & \frac{1}{\Gamma(\alpha)} \int_{0}^{t}(t-s)^{\alpha-1} M d s+2|m|+M_{1}+\frac{2}{\Gamma(\alpha+1)} \int_{0}^{1}(1-s)^{\alpha} M d s \\
\leq & \left(\frac{1}{\Gamma(\alpha+1)}+\frac{2}{\Gamma(\alpha+2)}\right) M+M_{1}+2|m|=A, \\
\|F(x)\|= & \max _{t \in[0,1]}|F(x)(t)| \leq\left(\frac{1}{\Gamma(\alpha+1)}+\frac{2}{\Gamma(\alpha+2)}\right) M+M_{1}+2|m|=A .
\end{aligned}
$$

Considering

$$
\begin{aligned}
F^{\prime}(x)(t)= & \frac{1}{\Gamma(\alpha-1)} \int_{0}^{t}(t-s)^{\alpha-2} f\left(s, x(s),{ }^{C} D^{\beta} x(s)\right) d s-2 m+2 y(x) \\
& +\frac{2}{\Gamma(\alpha+1)} \int_{0}^{1}(1-s)^{\alpha} f\left(s, x(s),{ }^{C} D^{\beta} x(s)\right) d s,
\end{aligned}
$$

and

$$
\begin{aligned}
& \int_{0}^{t}(t-s)^{-\beta} s^{\alpha-1} d s=t^{\alpha-\beta} B(\alpha, 1-\beta), \\
& B(\alpha, n-\beta)=\frac{\Gamma(\alpha) \Gamma(n-\beta)}{(\alpha-\beta+n-1) \Gamma(\alpha-\beta+n-1)},
\end{aligned}
$$

we obtain

$$
\begin{aligned}
{ }^{C} D^{\beta}(F x)(t)= & \frac{1}{\Gamma(1-\beta)} \int_{0}^{t}(t-s)^{-\beta}\left(\frac{1}{\Gamma(\alpha-1)} \int_{0}^{s}(s-\tau)^{\alpha-2} f\left(\tau, x(\tau),{ }^{C} D^{\beta} x(\tau)\right) d \tau-2 m\right. \\
& \left.+2 y(x)+\frac{2}{\Gamma(\alpha+1)} \int_{0}^{1}(1-\tau)^{\alpha} f\left(\tau, x(\tau),{ }^{C} D^{\beta} x(\tau)\right) d \tau\right) d s \\
= & \frac{1}{\Gamma(\alpha-\beta)} \int_{0}^{t}(t-s)^{\alpha-1-\beta} f\left(s, x(s),{ }^{C} D^{\beta} x(s)\right) d s+\frac{2 t^{1-\beta}}{\Gamma(2-\beta)}(y(x)-m) \\
& +\frac{2 t^{1-\beta}}{\Gamma(2-\beta) \Gamma(\alpha+1)} \int_{0}^{1}(1-s)^{\alpha} f\left(s, x(s),{ }^{C} D^{\beta} x(s)\right) d s
\end{aligned}
$$

thus

$$
\begin{aligned}
\left\|{ }^{C} D^{\beta}(F x)\right\| \leq & \frac{1}{\Gamma(\alpha-\beta)} \int_{0}^{t}(t-s)^{\alpha-1-\beta}\left|f\left(s, x(s),{ }^{C} D^{\beta} x(s)\right)\right| d s+\frac{2}{\Gamma(2-\beta)}(|y(x)|+|m|) \\
& +\frac{2}{\Gamma(2-\beta) \Gamma(\alpha+1)} \int_{0}^{1}(1-s)^{\alpha}\left|f\left(s, x(s),{ }^{C} D^{\beta} x(s)\right)\right| d s \\
\leq & \left(\frac{1}{\Gamma(\alpha-\beta+1)}+\frac{2}{\Gamma(2-\beta) \Gamma(\alpha+2)}\right) M+\frac{2}{\Gamma(2-\beta)}\left(M_{1}+|m|\right)=B .
\end{aligned}
$$


Hence, we can conclude that

$$
\|F x\|_{1}=\max \left\{\|F x\|,\left\|{ }^{C} D^{\beta}(F x)\right\|\right\} \leq r .
$$

From the expression of $(F x)(t)$ and ${ }^{C} D^{\beta}(F x)(t)$, it is easy to see that $(F x)(t) \in C[0,1]$, ${ }^{C} D^{\beta}(F x)(t) \in C[0,1]$. Consequently $F: E \rightarrow E$.

In what follows we show that $F$ is completely continuous.

(a) For each $t \in[0,1]$, we have

$$
\begin{aligned}
& \|F x\| \leq\left(\frac{1}{\Gamma(\alpha+1)}+\frac{2}{\Gamma(\alpha+2)}\right) M+M_{1}+2|m|=A, \\
& \left\|{ }^{C} D^{\beta}(F x)\right\| \leq\left(\frac{1}{\Gamma(\alpha-\beta+1)}+\frac{2}{\Gamma(2-\beta) \Gamma(\alpha+2)}\right) M+\frac{2}{\Gamma(2-\beta)}\left(M_{1}+|m|\right)=B,
\end{aligned}
$$

which shows that $F$ is uniform bounded.

(b) For each $t_{1}, t_{2} \in[0,1], t_{1}<t_{2}$, and it implies that

$$
\begin{aligned}
\left|F(x)\left(t_{2}\right)-F(x)\left(t_{1}\right)\right| & \\
\leq \mid & \frac{1}{\Gamma(\alpha)} \int_{0}^{t_{1}}\left[\left(t_{2}-s\right)^{\alpha-1}-\left(t_{1}-s\right)^{\alpha-1}\right] f\left(s, x(s),{ }^{C} D^{\beta} x(s)\right) d s \\
& +\frac{1}{\Gamma(\alpha)} \int_{t_{1}}^{t_{2}}\left(t_{2}-s\right)^{\alpha-1} f\left(s, x(s),{ }^{C} D^{\beta} x(s)\right) d s \mid \\
& +2\left(|m|+|y(x)|+\frac{1}{\Gamma(\alpha+1)} \int_{0}^{1}(1-s)^{\alpha}\left|f\left(s, x(s),{ }^{C} D^{\beta} x(s)\right)\right| d s\right)\left(t_{2}-t_{1}\right) \\
\leq & \frac{M}{\Gamma(\alpha)} \int_{0}^{t_{1}}\left[\left(t_{2}-s\right)^{\alpha-1}-\left(t_{1}-s\right)^{\alpha-1}\right] d s+\frac{M}{\Gamma(\alpha)} \int_{t_{1}}^{t_{2}}\left(t_{2}-s\right)^{\alpha-1} d s \\
& +\left(2|m|+2 M_{1}+\frac{2 M}{\Gamma(\alpha+2)}\right)\left(t_{2}-t_{1}\right) \\
= & \frac{M}{\Gamma(\alpha+1)}\left(t_{2}^{\alpha}-t_{1}^{\alpha}\right)+\left(2|m|+2 M_{1}+\frac{2 M}{\Gamma(\alpha+2)}\right)\left(t_{2}-t_{1}\right)
\end{aligned}
$$

and

$$
\begin{aligned}
\left|{ }^{C} D^{\beta}(F x)\left(t_{2}\right)-{ }^{C} D^{\beta}(F x)\left(t_{1}\right)\right| \leq \mid & \frac{1}{\Gamma(\alpha-\beta)} \int_{0}^{t_{2}}\left(t_{2}-s\right)^{\alpha-1-\beta} f\left(s, x(s),{ }^{C} D^{\beta} x(s)\right) d s \\
& -\frac{1}{\Gamma(\alpha-\beta)} \int_{0}^{t_{1}}\left(t_{1}-s\right)^{\alpha-1-\beta} f\left(s, x(s),{ }^{C} D^{\beta} x(s)\right) d s \mid \\
& +\frac{2}{\Gamma(2-\beta)}(|y(x)|+|m|)\left(t_{2}^{1-\beta}-t_{1}^{1-\beta}\right) \\
& +\frac{2\left(t_{2}^{1-\beta}-t_{1}^{1-\beta}\right)}{\Gamma(2-\beta) \Gamma(\alpha+1)} \int_{0}^{1}(1-s)^{\alpha}\left|f\left(s, x(s),{ }^{C} D^{\beta} x(s)\right)\right| d s \\
\leq & \frac{M}{\Gamma(\alpha-\beta+1)}\left(t_{2}^{\alpha-\beta}-t_{1}^{\alpha-\beta}\right)+\frac{2\left(M_{1}+|m|\right)}{\Gamma(2-\beta)}\left(t_{2}^{1-\beta}-t_{1}^{1-\beta}\right) \\
& +\frac{2 M}{\Gamma(\alpha+2) \Gamma(2-\beta)}\left(t_{2}^{1-\beta}-t_{1}^{1-\beta}\right) .
\end{aligned}
$$


The right-hand sides of equations (3.1) and (3.2) tend to zero when $t_{1} \rightarrow t_{2}$, so $F$ is compact as consequence of the Arzela-Ascoli theorem, and $F$ is continuous. We claim that $F$ is completely continuous. Combing the two steps above with lemma 2.3 , we deduce that the problem (1.1)-(1.3) has a solution on $E$.

Theorem 3.3 Assume that $f:[0,1] \times \mathbb{R} \times \mathbb{R} \rightarrow \mathbb{R}$ is continuous, $y:(C[0,1], \mathbb{R}) \rightarrow \mathbb{R}$ is continuous, and they satisfy

(H4) $|f(t, x, z)| \leq k(t)+c_{1}|x|^{\rho_{1}}+c_{2}|z|^{\rho_{2}}, x, z \in \mathbb{R}$

(H5) $|y(x)| \leq c_{3}\|x\|^{\rho_{3}}, x \in C([0,1], \mathbb{R})$;

where $k(t) \geq 0 \in L[0,1], 0<\rho_{i}<1$, and $c_{i} \geq 0$ for $i=1,2,3$. Then the BVP (1.1)-(1.3) has a solution.

Proof First, we define

$$
U=\left\{x: x \in X,\|x\|_{1} \leq R\right\},
$$

where

$$
\begin{aligned}
R \geq & \max \left\{\left(4 P c_{1}\right)^{\frac{1}{1-\rho_{1}}},\left(4 P c_{2}\right)^{\frac{1}{1-\rho_{2}}},\left(4 c_{3}\right)^{\frac{1}{1-\rho_{3}}},\right. \\
& \left.\left(4 Q c_{1}\right)^{\frac{1}{1-\rho_{1}}},\left(4 Q c_{2}\right)^{\frac{1}{1-\rho_{2}}},\left(\frac{8 c_{3}}{\Gamma(2-\beta)}\right)^{\frac{1}{1-\rho_{3}}}, 4 \delta, 4 \eta\right\}
\end{aligned}
$$

and

$$
\begin{aligned}
& P=\frac{1}{\Gamma(\alpha+1)}+\frac{2}{\Gamma(\alpha+2)}, \\
& Q=\frac{1}{\Gamma(\alpha-\beta+1)}+\frac{2}{\Gamma(2-\beta) \Gamma(\alpha+2)}, \\
& \delta=\frac{1}{\Gamma(\alpha)} \int_{0}^{t}(t-s)^{\alpha-1} k(s) d s+\frac{2}{\Gamma(\alpha+1)} \int_{0}^{1}(1-s)^{\alpha} k(s) d s+2|m|, \\
& \eta=\frac{1}{\Gamma(\alpha-\beta)} \int_{0}^{t}(t-s)^{\alpha-1-\beta} k(s) d s+\frac{2}{\Gamma(2-\beta) \Gamma(\alpha+1)} \int_{0}^{1}(1-s)^{\alpha} k(s) d s+\frac{2|m|}{\Gamma(2-\beta)} .
\end{aligned}
$$

Now we prove that $F: U \rightarrow U$. For any $x \in U$, we have

$$
\begin{aligned}
&\|F x\| \leq \frac{1}{\Gamma(\alpha)} \int_{0}^{t}(t-s)^{\alpha-1}\left|f\left(s, x(s),{ }^{C} D^{\beta} x(s)\right)\right| d s+2|m|+|y(x)| \\
&+\frac{2}{\Gamma(\alpha+1)} \int_{0}^{1}(1-s)^{\alpha}\left|f\left(s, x(s),{ }^{C} D^{\beta} x(s)\right)\right| d s \\
& \leq \delta+\frac{1}{\Gamma(\alpha)} \int_{0}^{t}(t-s)^{\alpha-1} d s\left(c_{1} R^{\rho_{1}}+c_{2} R^{\rho_{2}}\right)+c_{3} R^{\rho_{3}} \\
&+\frac{2}{\Gamma(\alpha+1)} \int_{0}^{1}(1-s)^{\alpha} d s\left(c_{1} R^{\rho_{1}}+c_{2} R^{\rho_{2}}\right) \\
& \leq \delta+\left(c_{1} R^{\rho_{1}}+c_{2} R^{\rho_{2}}\right) P+c_{3} R^{\rho_{3}}, \\
&\left\|{ }^{C} D^{\beta}(F x)\right\| \leq \frac{1}{\Gamma(\alpha-\beta)} \int_{0}^{t}(t-s)^{\alpha-1-\beta}\left|f\left(s, x(s),{ }^{C} D^{\beta} x(s)\right)\right| d s+\frac{2}{\Gamma(2-\beta)}(|y(x)|+|m|)
\end{aligned}
$$




$$
\begin{aligned}
& +\frac{2}{\Gamma(2-\beta) \Gamma(\alpha+1)} \int_{0}^{1}(1-s)^{\alpha}\left|f\left(s, x(s),{ }^{C} D^{\beta} x(s)\right)\right| d s \\
\leq & \eta+\frac{1}{\Gamma(\alpha-\beta)} \int_{0}^{t}(t-s)^{\alpha-1-\beta} d s\left(c_{1} R^{\rho_{1}}+c_{2} R^{\rho_{2}}\right)+\frac{2 c_{3} R^{\rho_{3}}}{\Gamma(2-\beta)} \\
& +\frac{2}{\Gamma(2-\beta) \Gamma(\alpha+1)} \int_{0}^{1}(1-s)^{\alpha} d s\left(c_{1} R^{\rho_{1}}+c_{2} R^{\rho_{2}}\right) \\
\leq & \eta+\left(c_{1} R^{\rho_{1}}+c_{2} R^{\rho_{2}}\right) Q+\frac{2 c_{3} R^{\rho_{3}}}{\Gamma(2-\beta)} .
\end{aligned}
$$

Considering $\|F x\|_{1}=\max \left\{\|F x\|,\left\|{ }^{C} D^{\beta}(F x)\right\|\right\}$, we can conclude that

$$
\begin{aligned}
\|F x\|_{1} & \leq \max \left\{\delta+\left(c_{1} R^{\rho_{1}}+c_{2} R^{\rho_{2}}\right) P+c_{3} R^{\rho_{3}}, \eta+\left(c_{1} R^{\rho_{1}}+c_{2} R^{\rho_{2}}\right) Q+\frac{2 c_{3} R^{\rho_{3}}}{\Gamma(2-\beta)}\right\} \\
& \leq \frac{R}{4}+\frac{R}{4}+\frac{R}{4}+\frac{R}{4} \leq R .
\end{aligned}
$$

Considering that $f, y$ are continuous functions, we take $M=\max _{t \in[0,1]}\left|f\left(t, x(t),{ }^{C} D^{\beta} x(t)\right)\right|$, $M_{1}=\max _{t \in[0,1]}|y(x(t))|$, and we can see that $F$ is completely continuous by considering the second step of Theorem 3.2.

As a consequence of Schauder's fixed-point theorem, we claim that the problem (1.1)-(1.3) has a solution on $U$.

\section{Examples}

In this section, in order to illustrate our results, we consider three examples.

Example 4.1 Consider the following boundary-value problem:

$$
\begin{aligned}
& { }^{C} D_{0+}^{1.5} x(t)=0.01 t x(t)+0.01 t^{2 C} D_{0+}^{0.5} x(t)+t, \\
& x(0)+x^{\prime}(0)=\sum_{i=1}^{n} c_{i} x\left(t_{i}\right), \quad \int_{0}^{1} x(t) d t=1, \\
& x^{\prime \prime}(0)=x^{\prime \prime \prime}(0)=\cdots=x^{(n-1)}(0)=0,
\end{aligned}
$$

where $0<t_{1}<t_{2}<\cdots<t_{n}<1, c_{i}, i=1,2, \ldots, n$, are given positive constants with $\sum_{i=1}^{n} c_{i}<\frac{1}{2}$.

Set $\alpha=1.5(n=2), \beta=0.5, f\left(t, x(t),{ }^{C} D_{0+}^{0.5} x(t)\right)=0.01 t x(t)+0.01 t^{2} C^{C} D_{0+}^{0.5} x(t)+t, y(x)=$ $\sum_{i=1}^{n} c_{i} x\left(t_{i}\right), m=1$. Let $t \in[0,1]$ and $x_{1}, x_{2}, y_{1}, y_{2} \in \mathbb{R}$,

$$
\begin{aligned}
\left|f\left(t, x_{1}, z_{1}\right)-f\left(t, x_{2}, z_{2}\right)\right| & =\left|0.01 t x_{1}-0.01 t x_{2}+0.01 t^{2} z_{1}+0.01 t^{2} z_{2}\right| \\
& \leq 0.01\left(\left|x_{1}-x_{2}\right|+\left|z_{1}-z_{2}\right|\right) .
\end{aligned}
$$

Hence the condition (H1) holds with $l=0.01$. Also we have

$$
\begin{aligned}
\left|y\left(x_{1}\right)-y\left(x_{2}\right)\right| & =\left|\sum_{i=1}^{n} c_{i} x_{1}\left(t_{i}\right)-\sum_{i=1}^{n} c_{i} x_{2}\left(t_{i}\right)\right| \\
& \leq \sum_{i=1}^{n} c_{i}\left\|x_{1}-x_{2}\right\| .
\end{aligned}
$$


Hence (H2) is satisfied with $l_{1}=\sum_{i=1}^{n} c_{i}<\frac{1}{2}$. As to (H3), we can show that

$$
\begin{aligned}
& \left(\frac{2}{\Gamma(\alpha+1)}+\frac{4}{\Gamma(\alpha+2)}\right) l+l_{1}=\left(\frac{2}{\Gamma(2.5)}+\frac{4}{\Gamma(3.5)}\right) \times 0.01+0.5 \simeq 0.5096<1, \\
& \frac{1}{\Gamma(2-\beta)}\left[\left(\frac{2}{\Gamma(\alpha)}+\frac{4}{\Gamma(\alpha+2)}\right) l+2 l_{1}\right] \simeq 0.76<1
\end{aligned}
$$

Then by Theorem 3.1, the problem (4.1)-(4.3) has a unique solution.

Example 4.2 Consider the following boundary-value problem:

$$
\begin{aligned}
& { }^{C} D_{0+}^{1.5} x(t)=(t-0.5)^{4}\left(x(t)^{\rho_{1}}+\left({ }^{C} D^{0.25} x(t)\right)^{\rho_{2}}\right), \\
& x(0)+x^{\prime}(0)=\left(t_{0}-0.5\right)^{4} x\left(t_{0}\right)^{\rho_{3}}, \quad \int_{0}^{1} x(t) d t=1, \\
& x^{\prime \prime}(0)=x^{\prime \prime \prime}(0)=\cdots=x^{(n-1)}(0)=0,
\end{aligned}
$$

where $\rho_{i}, t_{0}$ are given positive constants with $0<\rho_{i}, t_{0}<1$.

Set $\alpha=1.5(n=2), \beta=0.25, f\left(t, x(t),{ }^{C} D_{0+}^{0.25} x(t)\right)=(t-0.5)^{4}\left(x(t)^{\rho_{1}}+\left({ }^{C} D^{0.25} x(t)\right)^{\rho_{2}}\right), y(x)=$ $\left(t_{0}-0.5\right)^{4} x\left(t_{0}\right)^{\rho_{3}}, m=1$.

Note that

$$
\begin{aligned}
& |f(t, x, z)|=\left|(t-0.5)^{4}\left(x^{\rho_{1}}+z^{\rho_{2}}\right)\right| \leq \frac{1}{16}\left(|x|^{\rho_{1}}+|z|^{\rho_{2}}\right), \\
& |y(x)|=\left|\left(t_{0}-0.5\right)^{4} x\left(t_{0}\right)^{\rho_{3}}\right| \leq \frac{1}{16}\|x\|^{\rho_{3}} .
\end{aligned}
$$

Hence the conditions ( $\mathrm{H} 4)$ and (H5) hold with $k(t)=0, c_{1}=c_{2}=c_{3}=\frac{1}{16}$. Then by Theorem 3.3, the problem (4.4)-(4.6) has a solution.

Example 4.3 Consider the following boundary-value problem:

$$
\begin{aligned}
& { }^{C} D_{0+}^{1.5} x(t)=t+0.01 t x(t)^{\rho_{1}}+0.01 t^{2}\left({ }^{C} D^{0.25} x(t)\right)^{\rho_{2}}, \\
& x(0)=\left(t_{0}-0.5\right)^{4} x\left(t_{0}\right)^{\rho_{3}}, \quad \int_{0}^{1} x(t) d t=1, \\
& x^{\prime \prime}(0)=x^{\prime \prime \prime}(0)=\cdots=x^{(n-1)}(0)=0,
\end{aligned}
$$

where $\rho_{i}, t_{0}$ are given positive constants with $0<\rho_{i}, t_{0}<1$.

Set $\alpha=1.5(n=2), \beta=0.25, f\left(t, x(t),{ }^{C} D_{0+}^{0.25} x(t)\right)=t+0.01 t x(t)^{\rho_{1}}+0.01 t^{2}\left({ }^{C} D^{0.25} x(t)\right)^{\rho_{2}}$, $y(x)=\left(t_{0}-0.5\right)^{4} x\left(t_{0}\right)^{\rho_{3}}, m=1$.

Note that

$$
|f(t, x, z)| \leq 1+0.01\left(|x|^{\rho_{1}}+|z|^{\rho_{2}}\right), \quad|y(x)| \leq \frac{1}{16}\|x\|^{\rho_{3}} .
$$

Hence the conditions (H4) and (H5) hold with $k(t)=1, c_{1}=c_{2}=0.01, c_{3}=\frac{1}{16}$. Then by Theorem 3.3, the problem (4.7)-(4.9) has a solution. 


\section{Competing interests}

The authors declare that they have no competing interests.

\section{Authors' contributions}

The authors declare that the study was realized in collaboration with the same responsibility. All authors read and approved the final manuscript.

\section{Acknowledgements}

The authors sincerely thank the reviewers for their valuable suggestions and useful comments that have led to the present improved version of the original manuscript. This research is supported by the Natural Science Foundation of China (11071143, 61374074), Natural Science Outstanding Youth Foundation of Shandong Province (JQ201119) and supported by Shandong Provincial Natural Science Foundation (ZR2012AM009), also supported by Natural Science Foundation of Educational Department of Shandong Province (J11LA01).

\section{Received: 29 September 2013 Accepted: 23 December 2013 Published: 20 Jan 2014}

\section{References}

1. Oldham, KB, Spanier, J: The Fractional Calculus. Academic Press, New York (1974)

2. Kilbas, AA, Srivastava, HH, Trujillo, JJ: Theory and Applications of Fractional Differential Equations. Elsevier, Amsterdam (2006)

3. Zhang, S: The existence of a positive solution for nonlinear fractional differential equation. J. Math. Anal. Appl. 252, 804-812 (2000)

4. Zhang, S: Existence of positive solutions for some class of nonlinear fractional equation. J. Math. Anal. Appl. 278 $136-148(2003)$

5. Zhou, Y, Jiao, F, Li, J: Existence and uniqueness for p-type fractional neutral differential equations. Nonlinear Anal. 71, 2724-2733 (2009)

6. Sun, S, Li, Q, Li, Y: Existence and uniqueness of solutions for a coupled system of multi-term nonlinear fractional differential equations. Comput. Math. Appl. 64, 3310-3320 (2012)

7. Zhao, Y, Sun, S, Han, Z, Zhang, M: Positive solutions for boundary value problems of nonlinear fractional differential equations. Appl. Math. Comput. 217, 6950-6958 (2011)

8. Zhao, Y, Sun, S, Han, Z, Li, Q: Positive solutions to boundary value problems of nonlinear fractional differential equations. Abstr. Appl. Anal. 2011, Article ID 390543 (2011)

9. Zhao, Y, Sun, S, Han, Z, Li, Q: The existence of multiple positive solutions for boundary value problems of nonlinear fractional differential equations. Commun. Nonlinear Sci. Numer. Simul. 16, 2086-2097 (2011)

10. Zhao, Y, Sun, S, Han, Z, Li, Q: Theory of fractional hybrid differential equations. Comput. Math. Appl. 62, 1312-1324 (2011)

11. Feng, W, Sun, S, Han, Z, Zhao, Y: Existence of solutions for a singular system of nonlinear fractional differential equations. Comput. Math. Appl. 62, 1370-1378 (2011)

12. Bai, Z, Lü, H: Positive solutions for boundary value problem of nonlinear fractional differential equation. J. Math. Anal. Appl. 311, 495-505 (2005)

13. Zhang, S: Positive solutions for boundary-value problems of nonlinear fractional differential equations. Electron. J. Differ. Equ. 36, 1-12 (2006)

14. Sun, $S, Z$ Zhao, Y, Han, Z, Li, Y: The existence of solutions for boundary value problem of fractional hybrid differential equations. Commun. Nonlinear Sci. Numer. Simul. 17, 4961-4967 (2012)

15. Sun, S, Zhao, Y, Han, Z, Xu, M: Uniqueness of positive solutions for boundary value problems of singular fractional differential equations. Inverse Probl. Sci. Eng. 20, 299-309 (2012)

16. El-shahed, M, Nieto, JJ: Nontrivial solutions for a nonlinear multi-point boundary value problem of fractional order. Comput. Math. Appl. 59, 3438-3443 (2010)

17. Rehman, MU, Khan, RA: Existence and uniqueness of solutions for multi-point boundary value problems for fractional differential equations. Appl. Math. Lett. 23, 1038-1044 (2010)

18. Gallardo, JM: Second order differential operators with integral boundary conditions and generation of semigroups. Rocky Mt. J. Math. 30, 1265-1291 (2000)

19. Karakostas, GL, Tsamatos, P: Multiple positive solutions of some Fredholm integral equations arisen from nonlocal boundary-value problems. Electron. J. Differ. Equ. 30, 1-17 (2002)

20. Zhao, J, Wang, P, Ge, W: Existence and nonexistence of positive solutions for a class of third order BVP with integral boundary conditions in Banach spaces. Commun. Nonlinear Sci. Numer. Simul. 16, 402-413 (2011)

21. Wei, Z, Pang, C, Ding, Y: Positive solutions of singular Caputo fractional differential equations with integral boundary conditions. Commun. Nonlinear Sci. Numer. Simul. 17, 3148-3160 (2012)

22. Cabada, A, Wang, G: Positive solutions of nonlinear fractional differential equations with integral boundary value conditions. J. Math. Anal. Appl. 389, 403-411 (2012)

23. Chalishajar, DN, Karthikeyan, K: Existence and uniqueness results for boundary value problems of higher order fractional integro-differential equations involving Gronwall's inequality in Banach spaces. Acta Math. Sci. 33B, 758-772 (2013)

24. Rahman, MU, Khan, RA, Asif, NA: Three point boundary value problems for nonlinear fractional differential equations. Acta Math. Sci. 31B, 1337-1346 (2011)

25. Zhang, LH, Ahmad, B, Wang, GT, Agarwal, R: Nonlinear fractional integro-differential equations on unbounded domains in a Banach space. J. Comput. Appl. Math. 249, 51-56 (2013)

26. Li, N, Wang, CY: New existence results of positive solution for a class of nonlinear fractional differential equations. Acta Math. Sci. 33B, 847-854 (2013)

27. Anguraj, A, Karthikeyan, P, Rivero, M, Trujillo, JJ: On new existence results for fractional integro-differential equations with impulsive and integral conditions. Comput. Math. Appl. (2013). doi:10.1016/j.camwa.2013.01.034

28. Vong, SW: Positive solutions of singular fractional differential equations with integral boundary conditions. Math Comput. Model. 57, 1053-1059 (2013) 
29. Agarwal, RP, O'Regan, D, Stanek, S: Positive solutions for Dirichlet problems of singular nonlinear fractional differential equations. J. Math. Anal. Appl. 371, 57-68 (2010)

30. Benchohra, M, Hamani, S, Ntouyas, SK: Boundary value problems for differential equations with fractional order and nonlocal conditions. Nonlinear Anal. 71, 2391-2396 (2009)

10.1186/1687-1847-2014-25

Cite this article as: Yan et al.: Existence of solutions for fractional differential equations with integral boundary conditions. Advances in Difference Equations 2014, 2014:25

Submit your manuscript to a SpringerOpen ${ }^{\circ}$ journal and benefit from:

- Convenient online submission

- Rigorous peer review

- Immediate publication on acceptance

- Open access: articles freely available online

- High visibility within the field

- Retaining the copyright to your article

Submit your next manuscript at $\boldsymbol{s p r i n g e r o p e n . c o m ~}$ 